\title{
Gold in Thick-Film Conductors
}

\section{THE RELATIVE MERITS OF ALL-GOLD AND ALLOYED SYSTEMS}

\author{
R. H. Caley
}

The Electrical Research Association, Leatherhead, Surrey, England

\begin{abstract}
The growth of microelectronics since the arrival of the semiconductor has seen the emergence of several methods of interconnection, including thick-film technology. The number of thick-film conductors used for different assembly techniques has increased with the years, and this growth is typified by the extensive range of gold-containing conductor pastes now available. It is shown how the use of gold in conductor compositions is important to the extent that widely different assembly procedures can be performed on the same metallisation.
\end{abstract}

The invention of the transistor in 1948 transformed the electronics industry and signalled the beginnings of microelectronics. Subsequent improvements in semiconductor technology necessitated a complete reassessment of existing peripheral technologies so as to maximise the advantages offered by semiconductors. One major problem was how to make connections to semiconducting devices in a way that would realise their potential advantages. If successful techniques could be developed then the way was clear to more widespread improvements in connection methods to all components.

Several rival technologies emerged, including printed-circuit board, thin-film and thick-film technologies. The thick-film method, is perhaps, more flexible than the other two technologies, and this is particularly reflected in the range of thickfilm conductor materials available. Of these materials, gold-containing conductors provide the widest selection of properties to meet most needs of the engineer involved in component preparation and assembly.

\section{The Thick-Film Process}

The thick-film technique is basically a screen printing, drying and firing process. The method enables resistors, dielectric and conductor films to be deposited in any desired pattern on to flat ceramic substrates. Pastes suitable for screen printing are commercially available from over a dozen manufacturers. The compositions of these pastes include numerous combinations of metal, semiconducting oxide and glass powders, reactive oxide powders intended for bonding purposes, gelling agents, cellulose binders, and so on. In the case of conductor pastes, there are three principal constituents, namely a conductive pigment, a bonding agent and an organic vehicle.

Woven mesh screens are used in the printing operation. A pattern is delineated in emulsion covering each screen. Different screens and patterns are prepared for resistor, conductor and dielectric prints. A specially designed printer is used to deposit the patterned films on to the ceramic substrates. Prints are dried at temperatures around $120^{\circ} \mathrm{C}$ and then placed on a continuously moving belt passing horizontally through a furnace. Peak firing temperatures do not normally exceed $1000^{\circ} \mathrm{C}$ and in almost all cases the furnace atmosphere is air.

\section{The Range of Conductors in Use}

Clearly some restriction is imposed on the metals that can be used for the conductive constituent of thick-film conductors. The metal has to remain highly conductive after the firing process and metals showing undue oxidation cannot therefore be considered. Consequently, gold and silver are preferentially selected for conductor compositions either as the only component or in an alloy combination with other noble metals. Recently, systems using base metals have been devised in the hope of replacing some of the noble-metal systems, but most base-metal systems cannot be fired in air and there is therefore a reluctance to adopt their use because special atmosphere-controlled furnaces would be required. Also compatibility with resistor and dielectric systems will need to be established before base metals make a significant impact.

Conductor compositions can be loosely classified into two groups according to application; one group 


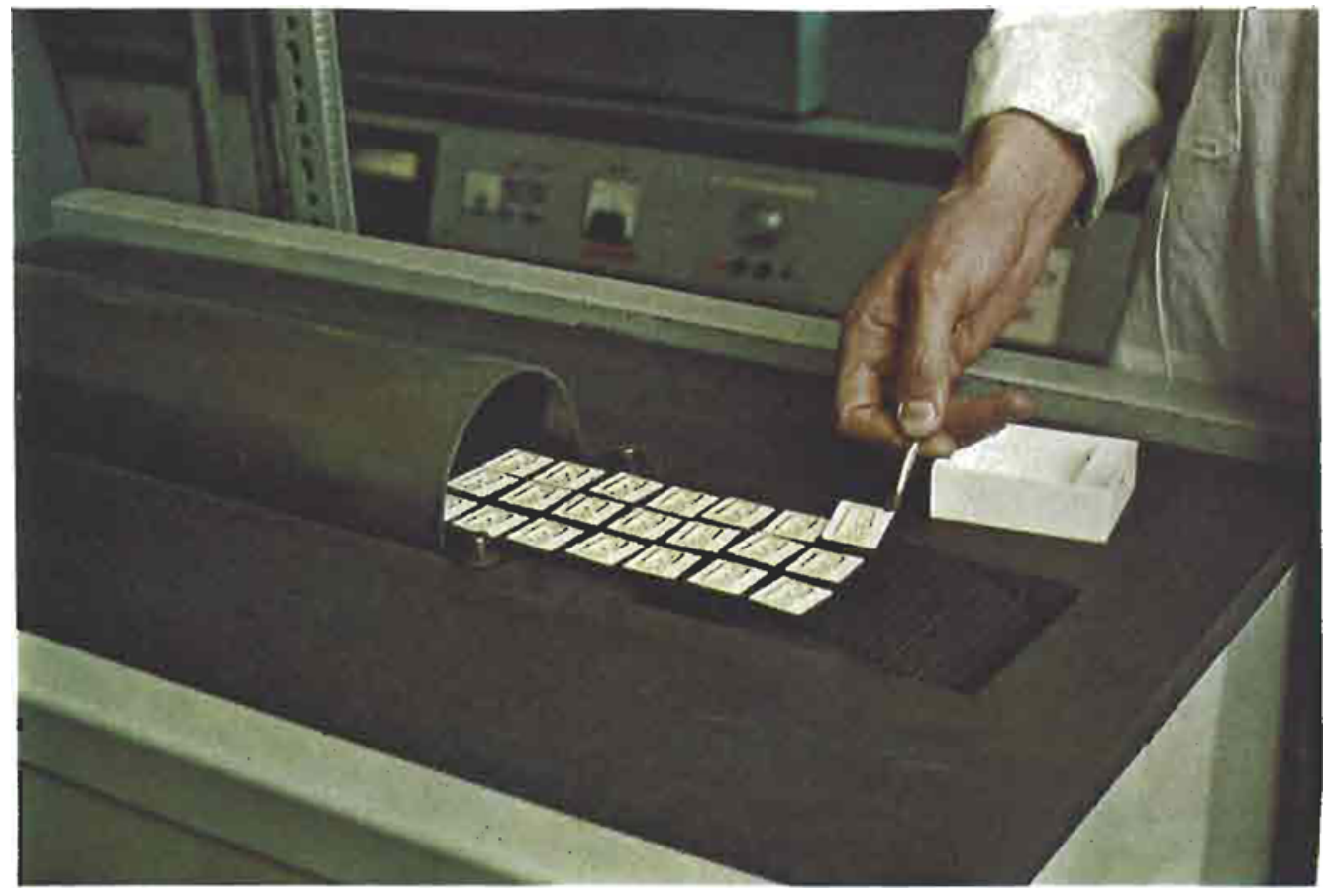

Thick-film gold conductors fired on to ceramic substrates emerging from a continuons belt furnace. The fring operation sinters the gold particles and promotes bonding either reactively hetween oxides in the film and the alumina of the ceramic or by glass bonding. Firing is usually in air, and pcak temperatures do not normally exceed $1000^{\circ} \mathrm{C}$

of conductors is widely used in the consumer field while the second group is employed in precision, high reliability applications such as in the computer area or aerospace work. The principal requisite of the first group is low cost and, therefore, silver-containing metallisations are suitable. The second group is satisfied by compositions based on gold since the use of silver is prohibited in certain high reliability applications because of its tendency to migrate in the presence of moisture or at elevated temperatures, particularly where high electric field strengths are present.

The gold-containing conductors may be further subdivided into four main groups, all-gold conductors, palladium-gold, platinum-gold and ternary alloy conductors.
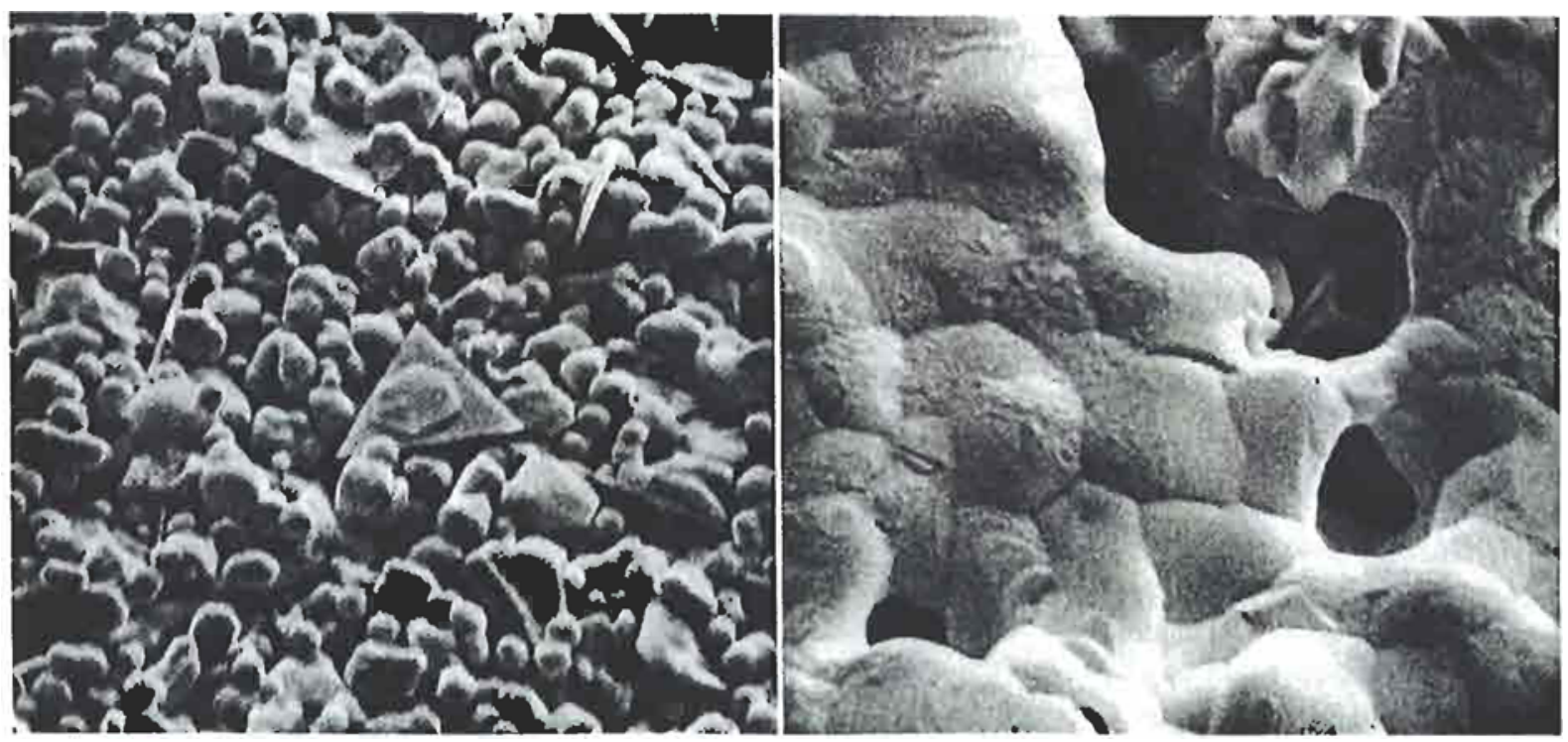

Scanning electron microscope photographs of gold conductors. On the left a dried print showing the variation of size and shape of the gold particles; on the right a fired film illustrating the densification and sintering of the gold particles $\times 5000$ 


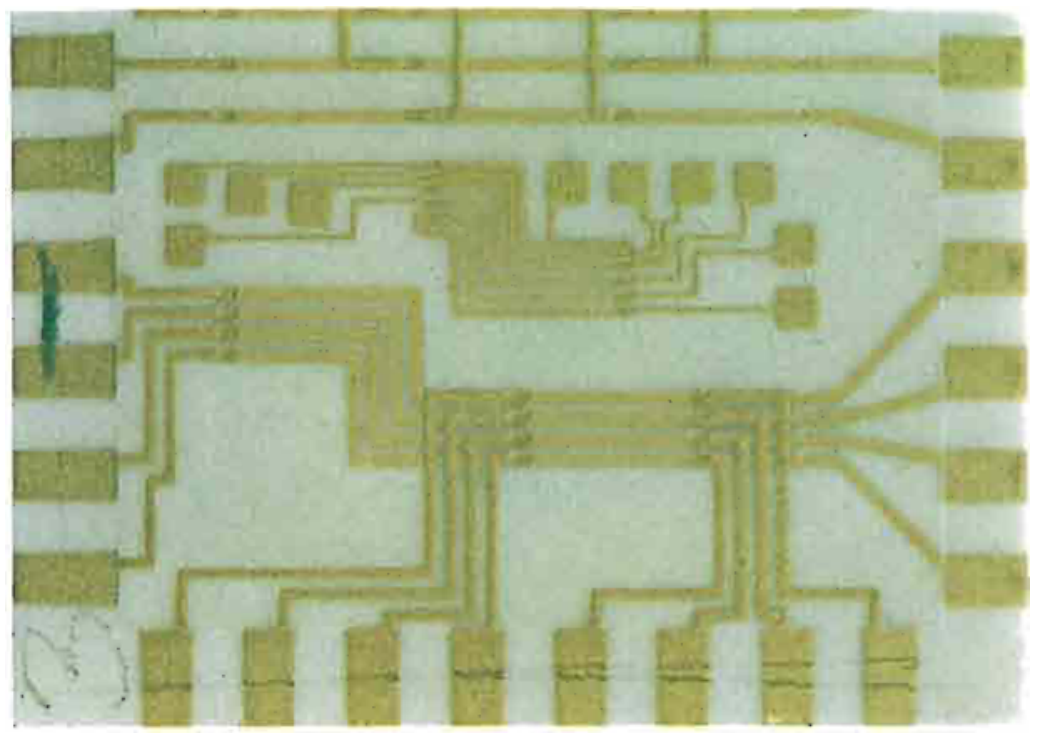

A partly completed multilayer structure using a thick-film gold for conductor layers separated by dielcetric. Openings (vias) are printed in the dielectric layer to interconneet the different conductor layers at various locations. Golds are preferred to other thick-film conductors because of their high conductivity, good stability and because they allow a wide choice of device bonding methods to be used

According to a recent count, ${ }^{*}$ sixty-two thick-film gold pastes are commercially available, reflecting their wide appeal and usage. This wide variety of compositions is the result of intense research and development which is constantly being undertaken to improve properties of pastes and fired metallisations.

\section{Constitution and Properties of Gold Conductors}

The principal requirement of any conductor is, of course, good electrical conductivity. Conductivity is controlled by several factors including the starting materials and firing profile. The gold powders present in these pastes vary from paste to paste. Scanning electron-microscope examinations of dried prints from a range of commercially available materials show a variety of particles sizes, shapes and distributions. Sizes are commonly in the range 1 to 10 microns. Shapes can be spherical, irregular or flakelike. Some dried prints contain small spheres only, whereas, other compositions show a predominance of flakes intermixed with micron-size spheres. It can be assumed that the observed differences between gold pastes reflect attempts by the manufacturers to improve densification during firing and hence conductivity and other properties.

The gold pastes contain certain additives for film adhesion purposes. Earlier gold compositions used glass as a bonding agent. During firing the glass flows to the film/ceramic interface, and on cooling, the glass layer provides a mechanical bond between film and ceramic.

Gold compositions over the past few years have been using certain oxide powders, including copper oxide, instead of glass for adhesion purposes. At

* Thick Film Conductor Survey, ERA Report 75-43. A review of this publication will be found on page 75 . peak firing temperatures, copper oxide reacts with the alumina or beryllia of the substrate, depending on its origin, and various compounds responsible for film adhesion are formed. Some reduction of the oxides within the film permits alloy formation between the gold and reduced oxide. In general, reactively-bonded golds have a higher adhesion than their glass counterparts when films are fired on highalumina (99.5 per cent) ceramics.

During the film-firing process, the organic materials burn off first at low temperatures and the films mature in the hottest zones of the furnace. The gold particles sinter together and densification of the film occurs. It has been suggested that the reactive oxide additions create alloy melting where the oxides contact gold particles and this melting assists densification.

\section{Electrical Conductivity}

The most electrically conductive thick-film golds have a conductivity well below that of the bulk metal. Even thick-film golds which are free from bonding agents of any description have resistivity values 40 per cent higher than the resistivity of bulk gold. Scanning electron microscopy confirms that fired gold films contain many porous regions accounting for the loss in conductivity.

\section{Thermocompression Bonding}

Thermocompression bonding of gold wires to gold conductors has been effectively performed for many years. Heat and pressure are simultaneously applied to wire and metallisation to promote interdiffusion of atoms across the area of contact. The end of the gold wire is flamed before bonding to produce a ball which is squashed during the application of pressure. This action increases the area of contact and leaves a strong bond. A smooth film surface is essential for a 
successful bond. The strength of wire to gold films bonds are generally acknowledged as being higher than the strength of bonds made to other thick-film conductors.

The additives contained in gold compositions for film adhesion sometimes cause thermocompression wire-bonding difficulties. Gold films containing glass often exhibit weakened adhesion after a wire bond has been fabricated. Undue bonding pressure cracks the glass joining the film to the ceramic and little effort is required to pull the metallisation away from the ceramic. The replacement of frit by certain oxides which react with the ceramic to provide film adhesion appears to have eliminated this fault, but a new fault has been observed with at least one reactively bonded composition. Whereas the glass in glass-bonded films falls to the ceramic during firing, the reactive oxides remain fairly homogeneously dispersed throughout the fired gold film. It has already been suggested that these oxides partially undergo some reduction on firing. Films stored for some time prove difficult or even impossible to wire bond to. It is thought that the impurity metals reoxidise at the surface in time, thus inhibiting good bonding.

\section{Ultrasonic Wire Bonding}

Ultrasonic wire bonding of aluminium wire to gold metallisations is less successful than thermocompression bonding because bonds aged for any length of time at elevated temperatures (e.g. $150^{\circ} \mathrm{C}$ ) deteriorate in strength. This ageing effect is attributed to the formation of brittle gold-aluminium intermetallic compounds.

If a wire bonding operation is required where heat has to be minimised, and the devices terminals are not of aluminium, then ultrasonic bonding of gold wire can be carried out, but the surface of the gold films has to be very smooth and clean.

\section{Eutectie Die Bonding}

Silicon devices require bonding to substrates for several reasons. Dies have to be anchored before wire bonding can take place on the active surface of the devices. It may also be convenient to make an electrical connection by way of the back of the chip and, therefore, back-bonding to a conductor on the substrate is an ideal solution. Finally, a direct contact to a metallisation may also be desirable for improved heat dissipation from the chip.

Eutectic die bonding allows silicon devices to be directly attached to gold films with or without prior metallisation of the reverse side of the chip. The process relies on the melting of the gold-silicon eutectic (17.5 per cent silicon) at a temperature of $370^{\circ} \mathrm{C}$. A silicon chip with its active side facing upwards is positioned on the gold metallisation and vibrated slightly by ultrasonics to remove any oxide coating on the silicon at the gold/silicon interface.

\section{Compatibility}

No incompatibility is reported between gold conductors and resistors or dielectrics as long as the same frit system in the conductors and resistors are used.

\section{Disadvantages of Gold Conductors}

Gold conductors suffer from two disadvantages. They are not routinely soldered by lead-tin solders on account of the fact that lead scavenges gold very rapidly. Solder activities with gold films are confined to the use of gold-tin solders or indium-based compositions in specialist applications.

The second disadvantage is that gold pastes are costly, regularly selling at around one hundred pounds sterling a troy ounce. Many users resent paying such prices and are continuously seeking cheaper substitutes. In the future, thick-film copper pastes could constitute a threat to the present-day usage of gold in several applications. On present performance, copper films have a slightly better conductivity than golds and are solderable with normal solders. On a performance as well as a cost basis, copper films could conceivably replace golds in applications where the volume of inter-connect material is high.

\section{Applications}

Gold conductors are very important for many applications where conductivity must be high, where line dimensions and the spaces between them are as low as three-thousandths of an inch (3 mils), and where thermocompression bonding techniques are to be used.

\section{Metallisation Carrier for Beam Leads}

Beam leads were introduced in the 1960's by Bell Laboratories to eliminate wire bonds between silicon device terminals and external metallisations. Using evaporation, electroplating and photolithographical techniques, gold beams can be built to extend beyond the edges of the chip and parallel to the device surface. The other ends of the beams make electrical contact with the device terminals. A special thermocompression bonding tool using a wobbling motion on the beams successively bonds each beam to the metallisation carrier located beneath the chip. Thus, electrical connections are completed at the same time as the chip is anchored to the substrate.

Formerly, thin-film golds were used as the carrier metallisation but the evaporation, plating and etching procedure is expensive compared to thick-film screening, assuming that the same results are achieved. 
(Beam leads cannot be bonded to non-gold metallisation). A fairly standard specification for beam leads is that the spacing between centres of beam leads is 10 mils. Most of the sixty-two gold pastes now available are capable of producing lines 5 mils wide separated by 5 mils spaces. Dimensions of 3 and 4 mils can also be obtained by careful screen preparation or by the alternative use of metal screens.

\section{Multilayers}

Conductors with fine-line properties and high conductivity are required for multilayer applications. Multilayer circuits are characterised by a high percentage of interconnections compared to other components. Thick films are ideally suited to prepare extensive interconnections without increasing circuit size significantly. Conductors are built upwards rather than outwards by printing and firing separate conductor and dielectric layers above each other, suitable openings being left in the dielectric prints for electrical connections to be made between conductor layers. For the circuit to remain compact, it is advantageous that line widths and spaces should be minimised wherever possible without risking "shorts" or "opens" since yield is an important factor with multilayers. However, such a narrowing of dimensions may increase line resistances beyond acceptable levels and the highest conductivity material is therefore selected. Additional requirements are that the conductor should not react with the dielectric during firing, and since most multilayers are used in high performance applications, silver migration must be avoided. Comparison of the conductors available reveals that gold conductors are currently best suited for multilayer work, although they may expect future competition from thick-film coppers.

\section{Microwaves}

Fine-line golds are increasingly used for microwave striplines, principally because gold has such a high conductivity compared with other materials. Narrow gaps between lines are essential in some microwave applications for optimum coupling, while film smoothness and cross-sectional rectangularity are both important since skin-depth considerations dictate that the bulk of film conductivity at microwave frequencies is confined to film extremities.

\section{Metallisation for Lead Frames}

Thermocompression bonding of lead frames to gold films printed at the edges of the substrate surface can also be carried out. The frames are prepared from cheaper materials such as nickel and the areas to be bonded are plated with a thin coating of gold. Much higher pressures are required compared to wire bonding pressures, and a special ram is needed. The process only works successfully with thick-film gold metallisations.

\section{Palladium-Gold Conductors}

During discussion of the properties of gold conductors it was indicated that the cost of pastes was high and that films could not be routinely soldered with lead-tin solders. Modifications to the all-gold systems go some way to alleviating these two disadvantages.

When a proportion of gold is replaced by palladium, the properties of films compared to gold systems alter significantly the amount by which properties can change being a function of the palladium-to-gold ratio. For instance, as the palladium content of palladium-gold compositions increases, the film conductivity is reduced. For maximum palladium contents, conductivity values are less than onehundreth of the conductivity of bulk gold.

Presently, substitution of palladium for gold reduces the cost of the conductor paste compared to all-gold compositions.

Probably the best advantage gained by the dilution of gold with palladium is that metallisations render themselves suitable for lead-tin soldering. Although the leach resistance of films improves as the palladium content increases, the resistance to scavenging by solder is not as a good as for other metallisations. A problem commonly experienced by all metallisations is that aged solder bonds weaken in strength. The palladium, gold and the tin of the solder combine to form an intermetallic compound that weakens bonding interfaces. Fortunately, final adhesion strengths in most cases are still tolerable for most applications.

The advantages achieved with palladium-golds have to be viewed against the sacrifices in certain properties attainable with gold. Beam-lead and leadframe bonding are precluded. Die bonding can only be satisfactorily performed with gold-silicon preforms placed between the die and palladium-gold. Wire bonds are less strong and the higher resistivity eliminates palladium-golds from many applications. While compatibility between gold conductors and resistors is satisfactory, high palladium-content conductors are reported to be incompatible with ruthenium resistors.

Only in specialist applications can palladium-golds be used instead of gold. If soldering performance is particularly critical and cost is less important, then platinum-golds should be considered.

\section{Platinum-Gold Conductors}

Platinum-golds generally provide better soldering properties compared with other thick-film conductors 
Platinum-golds are renowned for their exccllent soldering properties and they exhibit a widle compatibility with tlickfilm resistors. A resistor array is shown which uges a platinum-gold conductor for the interconnection of resistors and solder attachment of wires

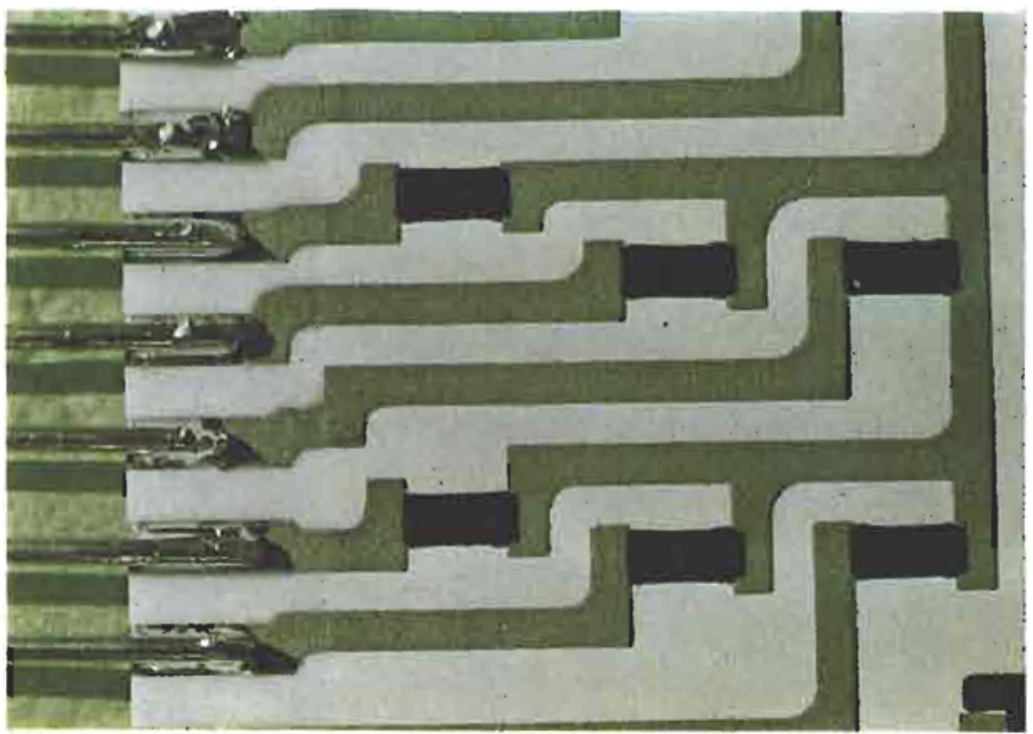

including palladium-silvers. The chicf disadvantage of platinum-golds is that they are sometimes more expensive than golds. Leach resistance of platinumgold is considerably better than that of palladiumgolds, and resistor compatibility is on a par with that shown by gold terminations. Otherwise the properties of platinum-golds closely resemble those of palladiumgolds including loss in strength of solder connections on ageing. Intermetallic compound formation is held responsible for much of the loss in strength, but this time the intermetallic is thought to be based on gold and tin.

\section{Ternary Noble Metal Conductors}

Several pastes are available claiming the use of three noble metal components instead of two metals. The pastes are basically platinum-golds or palladiumgolds where partial substitution of silver has been made for gold, or where mixtures of platinum and palladium with gold have been preferred. Properties are closely allied with their parent compositions.

\section{Summary}

Gold is an essential ingredient of thick-film conductors for applications requiring high performance and the integration of various components by wire, beam lead, or eutectic die bonding. Incorporation of a second component in gold films as exemplified by palladium-golds and platinum-golds improves the flexibility of conductors by allowing solder connections to be made. Moreover, platinum-gold offers the best soldering performance of any thick-film conductor. Although new materials, including base metals, may eventually gain a foothold in some applications, conductors using gold should continue to be used extensively for many more years.

\section{A Guide to Commercially Available Thick-Film Materials}

Thick-Film Conductor Survey: ERA Report 75-43

A very large number of thick-film conductor preparations is now available to the user, new and modified pastes having been introduced by a growing number of suppliers. These have made possible significant improvements in the quality of the end product and in its performance, but it has not always been possible to identify the advantages of improved adhesion, the virtues of alternative alloy compositions, better printing capability, and the introduction of reactive bonding.

To meet the need for a comprehensive guide to the thick-film conductors that are available commercially on a world-wide basis, this survey has been prepared to provide an objective and up-todate guide in one convenient volume.
The report opens with a concise account of the general principles underlying the selection of ingredients of pastes and of the compromises that have to be made to secure the desired characteristics. The properties of eight distinct groups of conductors are discussed, and the relative merits and disadvantages of each are summarised. Some 300 conductor pastes made by twelve manufacturers are listed under alloy-type headings, and the properties of each are set out in tabular form in terms of resistivity, solderability, leach resistance, adhesion, wire and die-bonding characteristics, printing capability, and compatibility with other materials.

The report, running to 196 pages, is available at $£ 45$ from ERA (formerly the Electrical Research Association), Leatherhead, Surrey, England. 